\title{
COMBINED TECHNIQUES OF CHEMICAL FIXATION AND CRYO- PRESERVATION USED TO REVEAL ULTRASTRUCTURAL DETAILS OF INTRACELLULAR PATHOGENS
}

\author{
Bryan T. Hansen*, Ted Hackstadt**, and Elizabeth R. Fischer** \\ * Western Washington University, 510 Nash Hall, Bellingham, WA 98225, **NIH/NIAID/ Rocky \\ Mountain Laboratories 903 South $4^{\text {th }}$ Street, Hamilton, MT 59840, U.S.A.
}

Cryo-preservation has long been regarded as the preferred technique for optimal preservation of structures in their native state, retaining components that would be lost or altered during conventional chemical fixations. [1] Viewing intracellular details of pathogens and their relationships to membranous compartments by cryo-SEM after high pressure freezing becomes difficult when fixed cytoplasm obscures the areas of interest. (Fig. 1) The goal of this study was to find a balance between preservation of structure and the ability to view more clearly the interaction of intracellular pathogens and their membrane bound compartments in tissue culture cells through the combined techniques of chemical fixation and cryo-fracturing for viewing by cryo-SEM.

The model system used for these experiments was cultured epithelial cells (HeLa) infected with chlamydiae. Cells were fixed with a minimum of $1 \%$ glutaraldehyde for 2 hours to address biosafety concerns. We first tested media that offered a broad range of cryoprotection to find a suitable buffer for reducing ice damage artifacts. Fig. 2 shows the results of cells that were suspended in either propanediol (a), HBSS + BSA (b), glycerol (c), dextran (d), distilled water with 5\% DMSO (e), or distilled water alone (f), and then plunge frozen in LN2, sublimated, fractured, coated and viewed using a Gatan Alto 2500 cryo-system on a Hitachi S-4500 FESEM. Cells suspended in water revealed the greatest structural depth for observation with the smoothest background consistency between cells.

Since glutaraldehyde was insufficient in maintaining the integrity of membrane structures during processing by plunge freezing, we investigated various fixation protocols that allowed for better preservation (Fig. 3). The conventional approach of glutaraldehyde followed by reduced osmium, and tannic acid (a) showed improvement over primary fixatives alone, but there was still considerable shrinkage of the bacteria and virtually no cytoplasmic detail. The addition of 1\% uranyl acetate overnight en bloc improved the appearance of the chlamydiae, but showed little improvement in cytoplasmic structures (b). In an effort to stabilize lipid components, we added malachite green to the primary fixative, and for greater cytoplasmic extraction went through two cycles of osmium and tannic acid prior to en bloc staining with uranyl acetate (c-e). [2] Results showed dramatic improvement in cell architecture. We tested this procedure on other tissue culture cells and pathogens (Fig. 4a \& b), and found similar success, suggesting these methods will be useful in studies of intracellular pathogens by cryo-SEM.

Reference:

[1] Kellenberger, E., Cryotechniques in Biological Microscopy, R.A. Steinbrecht and K. Zierold, eds., Springer-Verlag Berlin Heidelberg, 1987.

[2] Hayat, M.A., Stains and Cytochemical Methods, Plenum Press, New York, 1993. 


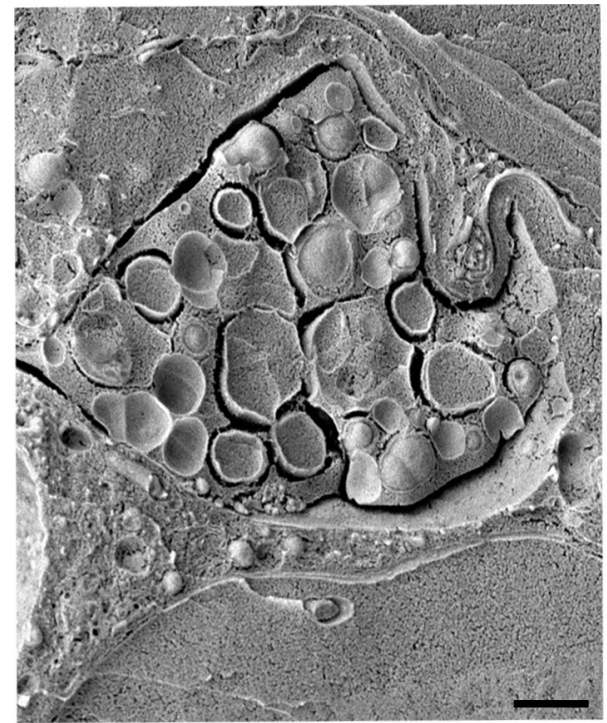

Fig. 1. High pressure frozen chlamydia infected HeLa cells. All scale bars $=1$ micron
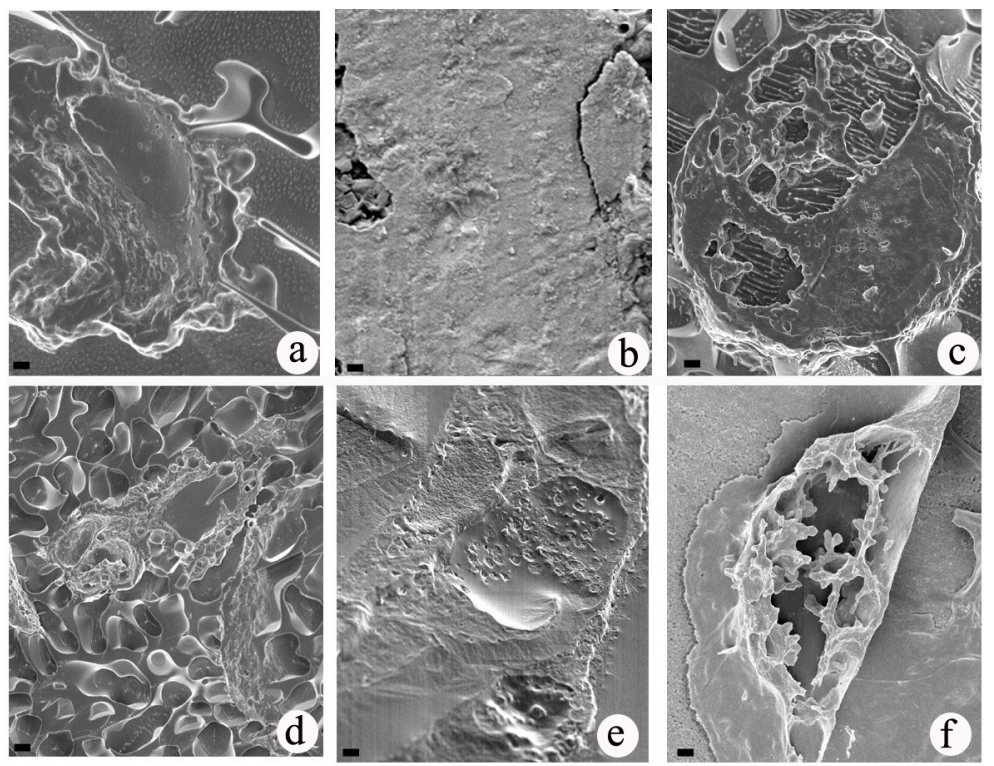

Fig.2. Cells suspended in either propanediol (a), HBSS + BSA (b), glycerol (c), dextran (d), distilled water with 5\% DMSO (e), or distilled water (f) prior to plunge freezing.
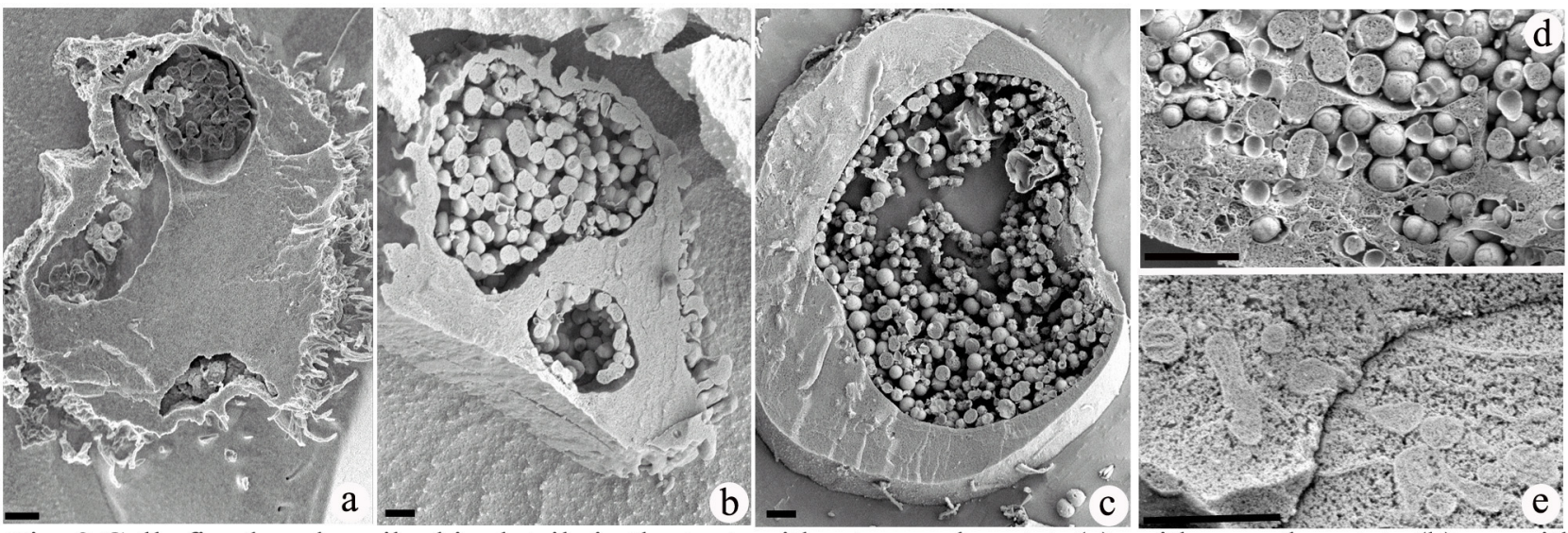

Fig. 3 Cells fixed as described in details in the text, without uranyl acetate(a), with uranyl acetate (b), or with malachite green added to the primary and multiple rounds of post-fixation steps (c-e).
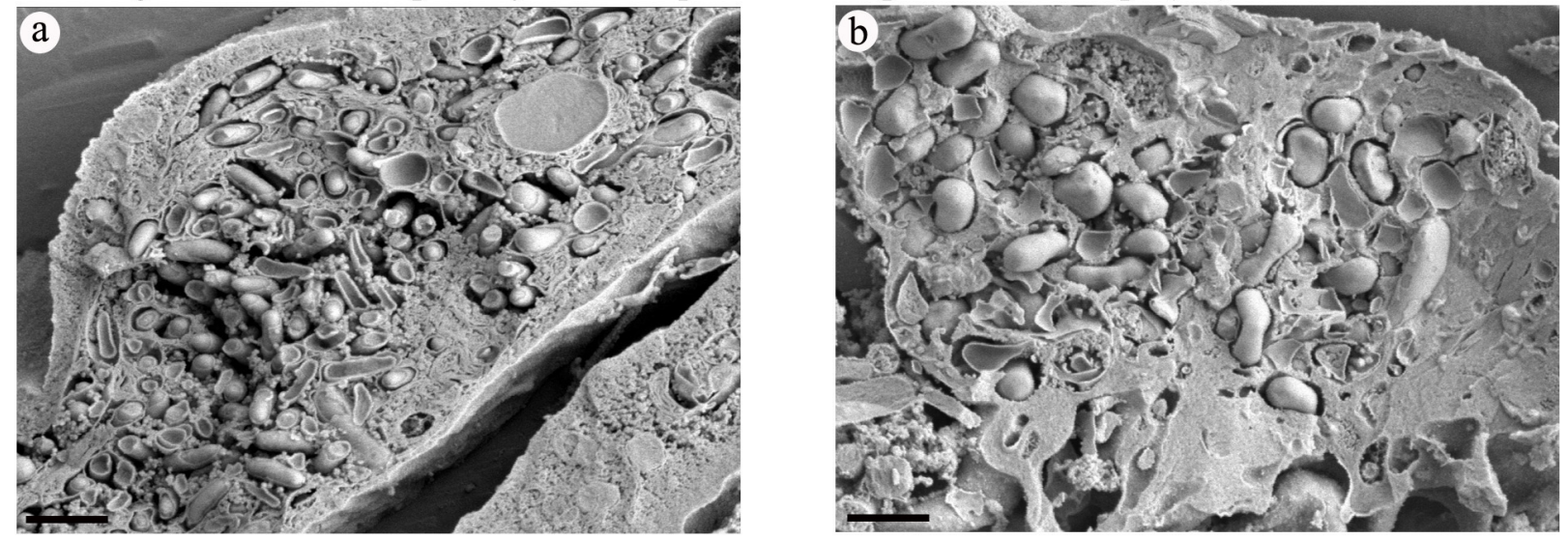

Fig. 4. Coxiella burnetii infected Vero cells (a) and Francisella tularensis infected mouse macrophages

(b) after chemical fixation, plunge freezing, fracturing, and viewing by cryo-SEM. 